Delft University of Technology

\title{
Cross-Correlated Contrast Source Inversion
}

Sun, Shilong; Kooij, Bert Jan; Jin, Tian; Yarovoy, Alexander G.

DOI

10.1109/TAP.2017.2673758

Publication date

2017

Document Version

Accepted author manuscript

Published in

IEEE Transactions on Antennas and Propagation

\section{Citation (APA)}

Sun, S., Kooij, B. J., Jin, T., \& Yarovoy, A. G. (2017). Cross-Correlated Contrast Source Inversion. IEEE

Transactions on Antennas and Propagation, 65(5), 2592 - 2603. https://doi.org/10.1109/TAP.2017.2673758

\section{Important note}

To cite this publication, please use the final published version (if applicable).

Please check the document version above.

\section{Copyright}

Other than for strictly personal use, it is not permitted to download, forward or distribute the text or part of it, without the consent of the author(s) and/or copyright holder(s), unless the work is under an open content license such as Creative Commons.

\section{Takedown policy}

Please contact us and provide details if you believe this document breaches copyrights.

We will remove access to the work immediately and investigate your claim. 


\title{
Cross-correlated Contrast Source Inversion
}

\author{
Shilong Sun, Bert Jan Kooij, Tian Jin, Member, IEEE, and Alexander G. Yarovoy, Fellow, IEEE
}

\begin{abstract}
In this paper, we improved the performance of the contrast source inversion (CSI) method by incorporating a socalled cross-correlated cost functional, which interrelates the state error and the data error in the measurement domain. The proposed method is referred to as the cross-correlated CSI (CCCSI). It enables better robustness and higher inversion accuracy than both the classical CSI and multiplicative regularized CSI (MRCSI). In addition, we show how the gradient of the modified cost functional can be calculated without significantly increasing the computational burden. The advantages of the proposed algorithms are demonstrated using a two-dimensional benchmark problem excited by a transverse magnetic (TM) wave as well as a transverse electric (TE) wave respectively, in comparison to classical CSI and MR-CSI.
\end{abstract}

Index Terms-Cross-correlated cost functional, contrast source inversion (CSI), finite difference frequency domain (FDFD), multiplicative regularized CSI (MR-CSI), transverse magnetic (TM), transverse electric (TE).

\section{INTRODUCTION}

$\mathbf{I}$ NVERSION techniques have been applied extensively in many fields, e.g. radar imaging [1], seismic imaging [2], medical imaging [3], [4], and so forth. Developments in inversion techniques and research are focused on computational efficiency, the incorporation of a priori information to circumvent computational artifacts, and the calibration to the real antenna radiating pattern especially in near-field scenarios [5], [6], [7]. Methods to solve the inverse scattering problems include non-iterative methods, e.g. linear sampling method [8], [9], and iterative methods [10]. The Contrast Source Inversion (CSI) method is an iterative frequency domain inversion method to retrieve the value of the contrast of scattering objects, which was first proposed by van den Berg et al. [10], and was later applied to subsurface object detection in combination with integral equations based on the Electric Field Integral Equation (EFIE) formulation, see Kooij et al. [11]. A priori information was introduced in the form of mathematical regularization constraints like the positivity constraints of the material properties and the Total Variation (TV) constraint in [12] to further enhance the performance. In [12], a multiplicative regularized CSI (MR-CSI) method is proposed, in which the estimation of the tuning parameter is avoided. In addition, Crocco et al. [13], [14], applied the socalled contrast source-extended Born-model to 2D subsurface scattering problems. Later the CSI technique was introduced in combination with the finite-difference frequency domain

Manuscript submitted Dec 02, 2015.

S. Sun, B. J. Kooij, and A. G. Yarovoy are with the Delft University of Technology, 2628 Delft, The Netherlands (e-mail: S.Sun@tudelft.nl; B.J.Kooij@ tudelft.nl; A.Yarovoy@tudelft.nl).

T. Jin is with the College of Electronic Science and Engineering, National University of Defense Technology, Changsha 410073, China (e-mail: tianjin@nudt.edu.cn).
(FDFD) scheme by Abubakar et al. [15], [16]. The scheme based on the FDFD technique turned out to have computational advantages compared to EFIE scheme, especially if a nonhomogeneous background, like the half-space configuration in GPR, is required in the inversion. For the aim of more accurately modelling any boundary shapes, finite-element method (FEM) was introduced and combined with CSI by Zakaria et al., and the 2D inversion results with the transverse magnetic (TM) wave and the transverse electric (TE) wave can be found in [17] and [18], respectively. FEM was applied as well to the contrast source-extended Born method by Attardo et al. [19].

One obvious drawback of the iterative methods is that a good initial guess must be provided beforehand to ensure the iterative inverting process converges to the global optimal solution. The reason is that there is more than one variable needed to be estimated during the inverting process. More specifically, the contrast and the contrast source are both unknown, and a less accurate initial guess is more likely to give a false gradient and thus leads the iterative inverting process to a local optimal solution. To overcome this drawback, the hybrid inversion schemes have been considered, which first recovered the shape of the scatterers faithfully by samplingtype technique, and then estimated their dielectric properties or improved the shape iteratively. The same idea can be found in the recently published papers [20], [21], [22], [23].

For iterative inversion methods, a cost function is normally needed which consists of the data error and the state error [24]. On one hand, it ensures that the algorithm fits the measurement data. On the other hand, it tends to optimize the estimation of the contrast and the contrast source to satisfy the Maxwell equations. In this paper, we show that a minor state error can become large when mapped into the measurement domain because of the ill-posedness of the inverse scattering problem. In another word, there might be cases in which both the state error and data error are minimized sufficiently, while the state error is still large when mapped into the measurement domain, which indicates that the estimated contrast is not the global optimal solution. Inspired by this fact, we introduce a new error equation that interrelates the state error and the data error in the measurement domain and modifies the cost functional accordingly. In doing so, the state error and the data error are cross-correlated, and the inverting process is stabilized by minimizing the state error not only in the field domain, but also in the measurement domain. We refer to the proposed algorithm as cross-correlated contrast source inversion (CCCSI) method. In addition, we also show how the gradient of the new cost functional can be calculated without significantly increasing the computational complexity. The performance of the proposed method is investigated based on a $2 \mathrm{D}$ benchmark problem excited by a TM-polarized wave and a TE-polarized wave, respectively. As can be observed 
from the results, the CCCSI method shows better robustness and higher inversion accuracy than classical CSI and MRCSI. Since the Maxwell's equations are formulated in a threedimensional FDFD formulation, it is already applicable to the reconstruction of future $3 \mathrm{D}$ scattering objects.

The remainder of the paper is organized as follows: Section II gives the problem statement and the introduction of classical CSI and MR-CSI. The cross-correlated CSI method is introduced in Section III. Simulation results based on a 2D benchmark problem are given in Section IV, in which the performance investigation of CCCSI in comparison to classical CSI and MR-CSI is fully discussed. Finally, we give our conclusions in Section V.

\section{Problem Statement and Classical CSi Based on FDFD}

\section{A. Problem statement}

We consider a scattering configuration as depicted in Figure 1 , which consists of a bounded, simply connected, in-

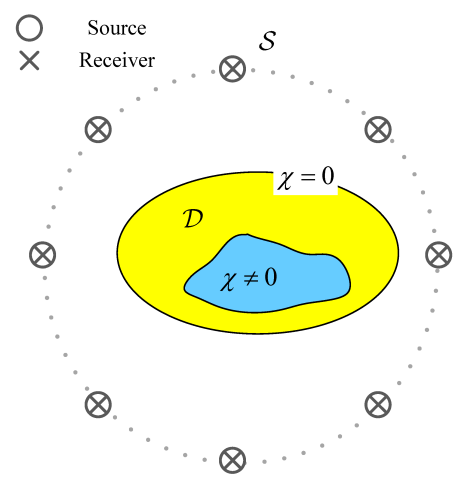

Fig. 1. The configuration of the inverse scattering problem.

homogeneous background domain $\mathcal{D}$, which is also referred to as field domain in this paper. The domain $\mathcal{D}$ contains an object, whose location and complex permittivity profile are unknown. The measurement domain $\mathcal{S}$ contains the sources and receivers. The sources are denoted by the subscript $p$ in which $p \in\{1,2,3 \ldots, P\}$, the receivers are denoted by the subscript $q$ in which $q \in\{1,2,3, \ldots, Q\}$. Sources and receivers that have equal subscripts are located at the same position. We use a right-handed coordinate system in which the unit vector in the invariant direction points out of the paper. The time factor of $\mathrm{e}^{\mathrm{i} \omega t}$ is considered in this paper.

In our notation for the vectorial quantities, we use a bold notation which represents a vector with three components. The general mathematical representations presented are consistent with any 3D configuration, in which the 2D TE and TM excitations are a special case, resulting in vectors containing zero elements. According to the linear relation among the incident electric field $\boldsymbol{E}_{p}^{i}$, the scattered electric field $\boldsymbol{E}_{p}^{\text {sct }}$ and the total electric fields $\boldsymbol{E}_{p}, \boldsymbol{E}_{p}^{\text {sct }}=\boldsymbol{E}_{p}-\boldsymbol{E}_{p}^{i}$, the scattering equation with respect to the scattered electric field $\boldsymbol{E}_{p}^{\text {sct }}$ can be easily obtained which is [25]

$$
\nabla \times \boldsymbol{\mu}^{-1} \nabla \times \boldsymbol{E}_{p}^{\mathrm{sct}}-\omega^{2} \boldsymbol{\epsilon}_{b} \boldsymbol{E}_{p}^{\mathrm{sct}}=\omega^{2} \boldsymbol{\chi} \boldsymbol{E}_{p},
$$

with $p=1,2, \ldots, P$. Here, $\boldsymbol{\mu}$ represents the permeability of the background and is assumed to be equal to the permeability of the free space in this paper; $\omega$ is the angular frequency; the permittivity $\varepsilon_{b}$ and the conductivity $\sigma_{b}$ of the background are incorporated into the complex permittivity $\epsilon_{b}$ satisfying $\epsilon_{b}=\varepsilon_{b}-\mathrm{i} \sigma_{b} / \omega$.

The scattering equation can be further formulated based on FDFD scheme by [25]

$$
\boldsymbol{A} \boldsymbol{e}_{p}^{\mathrm{sct}}=\omega^{2} \chi \boldsymbol{e}_{p}, \quad p=1,2, \ldots, P
$$

where $\boldsymbol{A}$ is the FDFD stiffness matrix; $\boldsymbol{e}_{p}^{\mathrm{sct}}$ and $\boldsymbol{e}_{p}$ are the scattered electric field and the total electric field in the form of a column vector, respectively; and $\chi$ is the contrast consisting of the difference of the permittivity $\varepsilon_{\mathrm{c}}$ and the difference of the conductivity $\sigma_{\mathrm{c}}$ with the relation of $\chi=\varepsilon_{\mathrm{c}}-\mathrm{i} \sigma_{\mathrm{c}} / \omega$. Then the solution of (2) is obtained by inverting the stiffness matrix $\boldsymbol{A}$, which yields $\boldsymbol{e}_{p}^{\mathrm{sct}}=\boldsymbol{A}^{-1} \omega^{2} \chi \boldsymbol{e}_{p}$. This leads to the data equation

$$
\boldsymbol{f}_{p}=\mathcal{M}_{\mathcal{S}} \boldsymbol{A}^{-1} \omega^{2} \chi \boldsymbol{e}_{p}, \quad \boldsymbol{x} \in \mathcal{S}, \quad p=1,2, \ldots, P,
$$

where $\mathcal{M}_{\mathcal{S}}$ is an operator that interpolates field values defined at the finite-difference grid points to the appropriate receiver positions.

In the remainder of this paper, $\omega^{2}$ is incorporated into $\boldsymbol{A}$ for the sake of conciseness. The inverse scattering problem is to reconstruct the contrast $\chi$ as a function of space from the incomplete measured field data $\left\{\boldsymbol{f}_{p}\right\}, p=1,2, \cdots, P$, which is full of challenges because of the nonlinearity and the ill-posedness.

\section{B. Classical CSI and MR-CSI}

Classical CSI is a method of iteratively minimizing a cost functional consisting of the data error and the state error for reconstructing the contrast source. The contrast is updated during the iterations. Specifically, the multiplication of the contrast $\chi$ and the total field $e_{p}$ is referred to as the contrast source, which is represented by $\boldsymbol{j}_{p}$. Then, the data error $\boldsymbol{\rho}_{p}$ and the state error $\gamma_{p}$ are defined by

$$
\boldsymbol{\rho}_{p}=\boldsymbol{f}_{p}-\mathcal{M}_{\mathcal{S}} \boldsymbol{A}^{-1} \boldsymbol{j}_{p}, \quad p=1,2, \ldots, P,
$$

and

$$
\gamma_{p}=\chi e_{p}^{\text {inc }}+\chi \mathcal{M}_{\mathcal{D}} \boldsymbol{A}^{-1} \boldsymbol{j}_{p}-\boldsymbol{j}_{p}, \quad p=1,2, \ldots, P,
$$

respectively. Here, $\mathcal{M}_{\mathcal{D}}$ is an operator that selects fields only inside the field domain $\mathcal{D}$. The cost functional is given by

$$
C_{\mathrm{CSI}}^{\boldsymbol{j}}\left(\boldsymbol{\chi}_{n-1}, \boldsymbol{j}_{p}\right)=\eta^{\mathcal{S}} \sum_{p=1}^{P}\left\|\boldsymbol{\rho}_{p}\right\|_{\mathcal{S}}^{2}+\eta^{\mathcal{D}} \sum_{p=1}^{P}\left\|\boldsymbol{\gamma}_{p}\right\|_{\mathcal{D}}^{2}
$$

with $\chi=\chi_{n-1}$. Here, $1 / \eta^{\mathcal{S}}=\sum_{p}\left\|\boldsymbol{f}_{p}\right\|_{\mathcal{S}}^{2}, 1 / \eta^{\mathcal{D}}=$ $\sum_{p}\left\|\chi e_{p}^{\mathrm{inc}}\right\|_{\mathcal{D}}^{2}$, and $\|\cdot\|_{\mathcal{S}}$ and $\|\cdot\|_{\mathcal{D}}$ represent the norms on the measurement space $L^{2}(\mathcal{S})$ and the field space $L^{2}(\mathcal{D})$, respectively. The contrast source $\boldsymbol{j}_{p}$ is iteratively optimized by minimizing the cost functional $C^{j}\left(\boldsymbol{\chi}, \boldsymbol{j}_{p}\right)$, followed by the 
update of the contrast $\chi$ which is done by minimizing the cost functional

$$
C_{\mathrm{CSI}}^{\boldsymbol{\chi}}\left(\boldsymbol{\chi}, \boldsymbol{j}_{p, n}\right)=\left.\eta^{\mathcal{D}} \sum_{p=1}^{P}\left\|\boldsymbol{\gamma}_{p}\right\|_{\mathcal{D}}^{2}\right|_{\boldsymbol{j}_{p}=\boldsymbol{j}_{p, n}} .
$$

MR-CSI is the CSI method regularized with a multiplicative weighted total variation (TV) constraint, which was first introduced by van den Berg et al. [12]. In comparison to CSI, the contrast $\chi$ in MR-CSI is now updated by minimizing the cost functional

$$
\begin{aligned}
& C_{\mathrm{MR}-\mathrm{CSI}}^{\boldsymbol{\chi}}\left(\boldsymbol{\chi}, \boldsymbol{j}_{p, n}\right)= \\
& C_{\mathrm{CSI}}^{\boldsymbol{j}}\left(\boldsymbol{\chi}, \boldsymbol{j}_{p}\right) \times\left.\frac{1}{V} \int_{\mathcal{D}} \frac{\|\nabla \boldsymbol{\chi}\|^{2}+\delta_{n-1}^{2}}{\left\|\nabla \boldsymbol{\chi}_{n-1}\right\|^{2}+\delta_{n-1}^{2}} d \boldsymbol{x}\right|_{\boldsymbol{j}_{p}=\boldsymbol{j}_{p, n}} .
\end{aligned}
$$

Here, $V=\int_{\mathcal{D}} d \boldsymbol{x}$, and $\delta_{n-1}^{2}$ are introduced for restoring the differentiability of the TV factor [12]. The value of $\delta_{n-1}^{2}$ is chosen to be large in the beginning of the optimization and small towards the end, which is given by

$$
\delta_{n-1}^{2}=C_{\mathrm{CSI}}^{\chi}\left(\chi_{n-1}, \boldsymbol{j}_{p, n}\right) \Delta^{2},
$$

where $\Delta$ denotes the mesh size of the discretized domain $\mathcal{D}$. It is worth noting that the contrast $\chi$ is assumed to be isotropic in this paper. Namely, for the TE case, only one component of $\chi$ is used in the TV penalty function. More details of classical CSI and MR-CSI can be found in [15], [25], [12], [26].

\section{CROSS-CORRELATED CSI}

\section{A. Motivation}

As aforementioned, a good initial guess is very critical for ensuring that the iterative methods can successfully converge to the global optimal solution. This can be explained firstly by the fact that there are two unknown variables - the contrast and the contrast source. The less accurate the initial guess is, the more inaccurate the gradient with respect to the contrast source will be. Secondly, although classical CSI is able to minimize the data error $\rho\left(\boldsymbol{j}_{p}\right)$ by constraining the state error $\gamma\left(\boldsymbol{\chi}, \boldsymbol{j}_{p}\right)$ at the same time, a global optimal solution can still not be guaranteed because of the ill-posedness of the inverse scattering problem. For simplicity, let us first define the measurement matrix as $\Phi:=\mathcal{M}_{\mathcal{S}} \boldsymbol{A}^{-1}$. The condition number of matrix $\boldsymbol{\Phi}$ is further defined as

$$
\kappa(\boldsymbol{\Phi}):=\frac{\sigma_{\max }(\boldsymbol{\Phi})}{\sigma_{\min }(\boldsymbol{\Phi})} .
$$

where $\sigma_{\max }(\boldsymbol{\Phi})$ and $\sigma_{\min }(\boldsymbol{\Phi})$ are maximal and minimal singular values of $\boldsymbol{\Phi}$, respectively. As discussed in Subsection IV-B, the measurement matrix has a large condition number, which means a minor state error in the field space $L^{2}(\mathcal{D})$ may cause a large error in the measurement space $L^{2}(\mathcal{S})$. This potential mismatch cannot be reflected by the cost functional of the classical CSI method. Inspired by this fact, we came up with the idea of introducing the so-called cross-correlated cost functional.

\section{B. Cross-correlated CSI}

In this subsection, a new cost functional is proposed, which interrelates the mismatch of the state equation and the data error in the measurement space. This proposed algorithm is referred to as the cross-correlated contrast source inversion method.

Specifically, the cross-correlated error $\boldsymbol{\xi}_{p}$ is defined as

$$
\boldsymbol{\xi}_{p}=\boldsymbol{f}_{p}-\boldsymbol{\Phi}\left(\chi \boldsymbol{e}_{p}^{\mathrm{inc}}+\chi \mathcal{M}_{\mathcal{D}} \boldsymbol{A}^{-1} \boldsymbol{j}_{p}\right) .
$$

Note that if the state error $\gamma_{p}$ is zero, then theoretically we have $\boldsymbol{\rho}_{p}=\boldsymbol{\xi}_{p}$. In classical CSI, sufficiently minimizing the cost function of Eq. (6) does not necessarily mean that the cross-correlated error is sufficiently minimized. Therefore, the cost functional of the contrast source in the proposed CCCSI method is modified and defined as

$$
\begin{aligned}
& C_{\mathrm{CCCSI}}^{j}\left(\boldsymbol{\chi}_{n-1}, \boldsymbol{j}_{p}\right)=\eta^{\mathcal{S}} \sum_{p=1}^{P}\left\|\boldsymbol{\rho}_{p}\right\|_{\mathcal{S}}^{2}+ \\
& \eta^{\mathcal{D}} \sum_{p=1}^{P}\left\|\boldsymbol{\gamma}_{p}\right\|_{\mathcal{D}}^{2}+\left.\eta^{\mathcal{S}} \sum_{p=1}^{P}\left\|\boldsymbol{\xi}_{p}\right\|_{\mathcal{S}}^{2}\right|_{\boldsymbol{\chi}=\boldsymbol{\chi}_{n-1}} .
\end{aligned}
$$

Subsequently, the gradient (Fréchet derivative) of the modified cost functional with respect to the contrast source $\boldsymbol{j}_{p}$ becomes

$$
\begin{aligned}
\boldsymbol{g}_{p, n}= & -2 \eta^{\mathcal{S}} \boldsymbol{\Phi}^{H} \boldsymbol{\rho}_{p}+2 \eta^{\mathcal{D}}\left(\boldsymbol{\chi \mathcal { M } _ { \mathcal { D } }} \boldsymbol{A}^{-1}-\boldsymbol{I}\right)^{H} \gamma_{p}- \\
& \left.2 \eta^{\mathcal{S}}\left(\boldsymbol{\Phi} \boldsymbol{\chi} \mathcal{M}_{\mathcal{D}} \boldsymbol{A}^{-1}\right)^{H} \boldsymbol{\xi}_{p}\right|_{\boldsymbol{\chi}=\boldsymbol{\chi}_{n-1}, \boldsymbol{j}_{p}=\boldsymbol{j}_{p, n-1}} .
\end{aligned}
$$

Here, $\boldsymbol{I}$ represents the identity matrix, and $(\cdot)^{H}$ is the conjugate transpose operator. Now suppose $\boldsymbol{j}_{p, n-1}$ and $\chi_{n-1}$ are known, then we update $\boldsymbol{j}_{p}$ by

$$
\boldsymbol{j}_{p, n}=\boldsymbol{j}_{p, n-1}+\alpha_{p, n} \boldsymbol{\nu}_{p, n},
$$

where $\alpha_{p, n}$ is constant and the update directions $\boldsymbol{\nu}_{p, n}$ are functions of the position. The update directions are chosen to be the Polak-Ribière conjugate gradient directions, which are given by

$$
\begin{aligned}
& \boldsymbol{\nu}_{p, 0}=0 \\
& \boldsymbol{\nu}_{p, n}=\boldsymbol{g}_{p, n}+\frac{\sum_{p^{\prime}}\left\langle\boldsymbol{g}_{p^{\prime}, n}, \boldsymbol{g}_{p^{\prime}, n}-\boldsymbol{g}_{p^{\prime}, n-1}\right\rangle_{\mathcal{D}}}{\sum_{p^{\prime}}\left\|\boldsymbol{g}_{p^{\prime}, n-1}\right\|_{\mathcal{D}}^{2}} \boldsymbol{\nu}_{p, n-1} n \geq 1,
\end{aligned}
$$

where $\langle\cdot\rangle_{\mathcal{D}}$ represents the inner product defined in the field space $L^{2}(\mathcal{D})$. The step size $\alpha_{p, n}$ can be explicitly found by minimizing the cost functional $C_{\mathrm{CCCSI}}^{j}\left(\chi_{n-1}, \boldsymbol{j}_{p, n-1}+\right.$ $\left.\alpha_{p, n} \boldsymbol{\nu}_{p, n}\right)$ (see Appendix A for the derivation).

Once the contrast source $\boldsymbol{j}_{p, n}$ is determined, we update the contrast $\chi$ by minimizing the cost functional of the contrast which is defined by

$$
C_{\text {CCCSI }}^{\chi}\left(\boldsymbol{\chi}, \boldsymbol{j}_{p, n}\right)=\eta^{\mathcal{D}} \sum_{p=1}^{P}\left\|\boldsymbol{\gamma}_{p}\right\|_{\mathcal{D}}^{2}+\eta^{\mathcal{S}} \sum_{p=1}^{P}\left\|\boldsymbol{\xi}_{p}\right\|_{\mathcal{S}}^{2}
$$

with $\boldsymbol{j}_{p}=\boldsymbol{j}_{p, n}$. Specifically, $\boldsymbol{\chi}$ is updated via

$$
\chi_{n}=\chi_{n-1}+\beta_{n} \boldsymbol{\nu}_{\boldsymbol{\chi}, n}
$$


where $\beta_{n}$ is constant and the update directions $\boldsymbol{\nu}_{p, n}$ are chosen to be the Polak-Ribière conjugate gradient directions, which are given by

$$
\begin{aligned}
& \boldsymbol{\nu}_{\boldsymbol{\chi}, 0}=0 \\
& \boldsymbol{\nu}_{\boldsymbol{\chi}, n}=\boldsymbol{g}_{\boldsymbol{\chi}, n}+\frac{\left\langle\boldsymbol{g}_{\boldsymbol{\chi}, n}, \boldsymbol{g}_{\boldsymbol{\chi}, n}-\boldsymbol{g}_{\boldsymbol{\chi}, n-1}\right\rangle_{\mathcal{D}}}{\left\|\boldsymbol{g}_{\boldsymbol{\chi}, n-1}\right\|_{\mathcal{D}}^{2}} \boldsymbol{\nu}_{\boldsymbol{\chi}, n-1} n \geq 1,
\end{aligned}
$$

where $\boldsymbol{g}_{\boldsymbol{\chi}, n}$ is the preconditioned gradient of the contrast cost functional $C_{\mathrm{CCCSI}}^{\chi}(\chi)$ defined as

$$
\boldsymbol{g}_{\boldsymbol{\chi}, n}=\frac{2 \eta^{\mathcal{D}} \sum_{p=1}^{P} \overline{\boldsymbol{e}_{p, n}} \gamma_{p}-2 \eta^{\mathcal{S}} \sum_{p=1}^{P} \overline{\boldsymbol{e}_{p, n}} \boldsymbol{\Phi}^{H} \boldsymbol{\xi}_{p}}{\sum_{p=1}^{P}\left|\boldsymbol{e}_{p, n}\right|^{2}}
$$

with $\chi=\chi_{n-1}, \boldsymbol{j}_{p}=\boldsymbol{j}_{p, n}$, where $\overline{(\cdot)}$ represents the conjugate operator. The step size $\beta_{n}$ is determined by minimizing the cost function

$$
\begin{gathered}
C_{\text {CCCSI }, n}^{\boldsymbol{\chi}}=\frac{\sum_{p=1}^{P}\left\|\left(\boldsymbol{\chi}_{n-1}+\beta_{n} \boldsymbol{\nu}_{\boldsymbol{\chi}, n}\right) \boldsymbol{e}_{p, n}-\boldsymbol{j}_{p, n}\right\|_{\mathcal{D}}^{2}}{\sum_{p=1}^{P}\left\|\left(\boldsymbol{\chi}_{n-1}+\beta_{n} \boldsymbol{\nu}_{\boldsymbol{\chi}, n}\right) \boldsymbol{e}_{p}^{\mathrm{inc}}\right\|_{\mathcal{D}}^{2}}+ \\
\eta^{\mathcal{S}} \sum_{p=1}^{P}\left\|\boldsymbol{f}_{p}-\boldsymbol{\Phi}\left(\boldsymbol{\chi}_{n-1}+\beta_{n} \boldsymbol{\nu}_{\boldsymbol{\chi}, n}\right) \boldsymbol{e}_{p, n}\right\|_{\mathcal{S}}^{2}
\end{gathered}
$$

This is a problem of finding the minimum of a single-variable function, and can be solved efficiently by the Brent's method [27], [28].

The CCCSI method is given in Algorithm 1, where $\Re\{\cdot\}$ represents the real part operator and, correspondingly, the imaginary part operator is represented by $\Im\{\cdot\}$. Since $\mathcal{M}_{\mathcal{D}}$ always exists together with the stiffness matrix $\boldsymbol{A}$, it is neglected for better readability in the remainder of this paper. It is worth noting that $\chi$ is assumed to be isotropic in this paper. Therefore, we average the two components of the contrast $\chi$ for TE case, and the three components of the contrast $\chi$ for 3D case, after each update of $\chi$.

\section{Initialization}

If no a priori information about the objects is available, the contrast sources are initialized by (see [29], [10])

$$
\boldsymbol{j}_{p, 0}=\frac{\left\|\boldsymbol{\Phi}^{H} \boldsymbol{f}_{p}\right\|_{\mathcal{D}}^{2}}{\left\|\boldsymbol{\Phi} \boldsymbol{\Phi}^{H} \boldsymbol{f}_{p}\right\|_{\mathcal{S}}^{2}} \boldsymbol{\Phi}^{H} \boldsymbol{f}_{p}
$$

which are obtained by back-propagation, multiplied by a weight to ensure that the data error is minimized. The contrast is initialized by (see [12])

$$
\chi_{0}=\frac{\sum_{p=1}^{P} \boldsymbol{j}_{p, 0} \overline{\boldsymbol{e}_{p, 0}}}{\sum_{p=1}^{P} \boldsymbol{e}_{p, 0} \overline{\boldsymbol{e}_{p, 0}}},
$$

with $\boldsymbol{e}_{p, 0}=\boldsymbol{e}_{p}^{\mathrm{inc}}+\mathcal{M}_{\mathcal{D}} \boldsymbol{A}^{-1} \boldsymbol{j}_{p, 0}$.

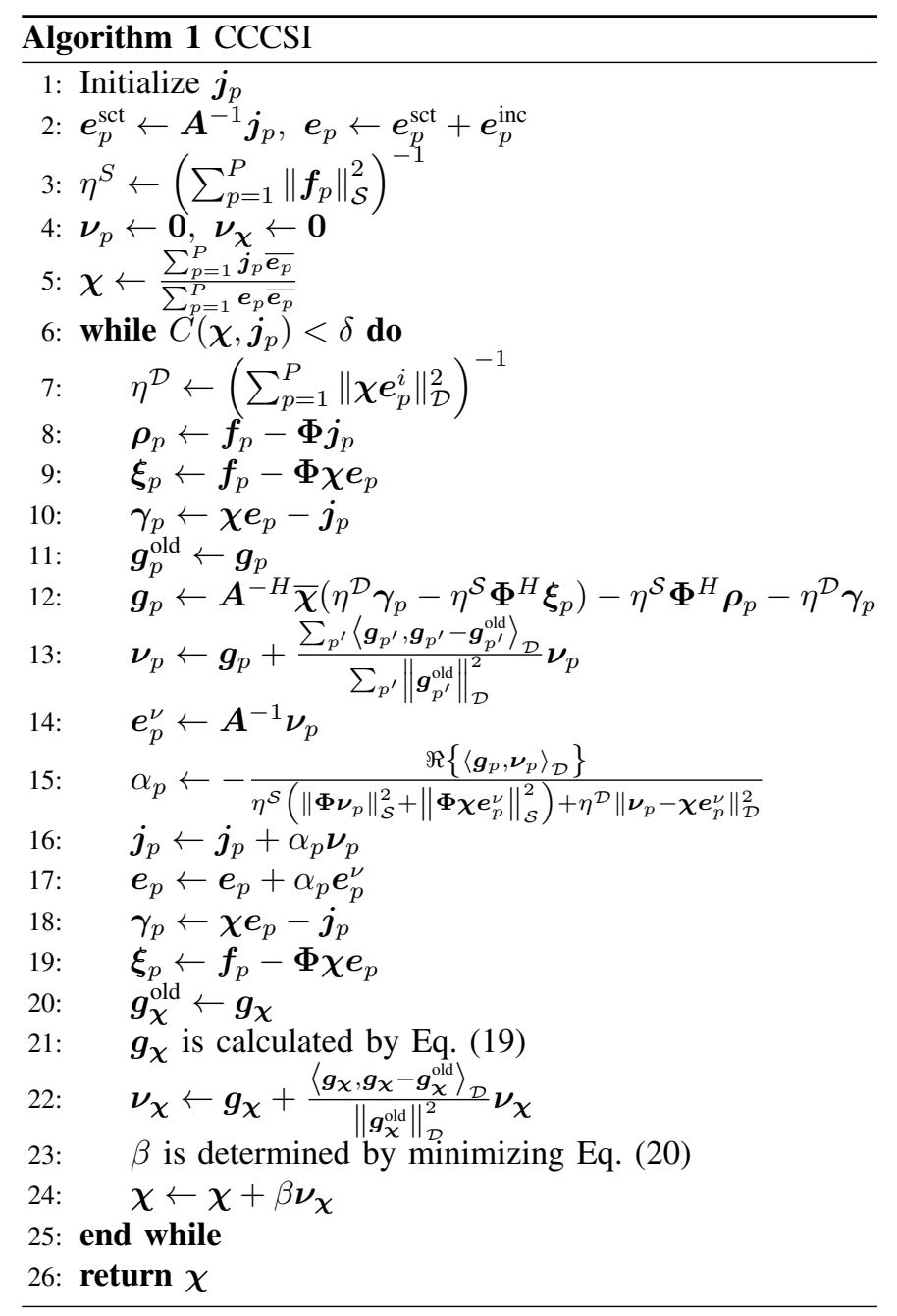

\section{Computational complexity}

In this subsection, we show that the CCCSI method can be implemented without significantly increasing the computational complexity compared to the classical CSI method. Note that since the selecting matrix $\mathcal{M}_{\mathcal{S}} \in \mathbb{C}^{M \times N}$ has only $M<<N$ rows, the matrix $\boldsymbol{\Phi}$ can be calculated iteratively by solving $M$ linear systems of equations,

$$
\boldsymbol{A}^{T} \boldsymbol{\varphi}_{m}=\mathcal{M}_{\mathcal{S}}^{m T}, \quad m=1,2, \ldots, M
$$

where, $\mathcal{M}_{\mathcal{S}}^{m}$ is the $m^{t h}$ row of the selecting matrix $\mathcal{M}_{\mathcal{S}}$, and $(\cdot)^{T}$ represents the transpose operator. The matrix $\boldsymbol{\Phi}$ is assembled by $\boldsymbol{\Phi}=\left[\boldsymbol{\varphi}_{1}, \boldsymbol{\varphi}_{2}, \ldots, \boldsymbol{\varphi}_{M}\right]^{T}$. Since $\boldsymbol{\Phi}$ has only $M<<N$ rows, it is computationally much more efficient than the LU decomposition of the stiffness matrix $\boldsymbol{A}$ (if we use LU decomposition). This feature makes it suitable to be computed and stored beforehand, which is of great importance for real applications, especially for 3D inverse scattering problems. Although CCCSI requires more matrix-vector multiplications, the extra computational cost is not significant by noting that the matrix $\boldsymbol{\Phi}$ has only $M<<N$ rows. This is further demonstrated in Subsection IV-E. 


\section{Simulation RESUlts}

\section{A. Configuration}

In this section, the proposed algorithm is tested with a $2 \mathrm{D}$ benchmark problem - the "Austria" profile, which was also used by Belkebir and Tijhuis [30], Litman et al. [31], and Berg et al. [26], [32]. Based on the benchmark problem, the performance of CCCSI is analyzed in comparison to classical CSI and MR-CSI.

Specifically, the objects to be inverted consist of two disks and one ring. Let us first establish our coordinate system such that the $z$-axis is parallel to the axis of the objects. The disks of radius $0.2 \mathrm{~m}$ are centred at $(-0.3,0.6) \mathrm{m}$ and $(0.3,0.6) \mathrm{m}$. The ring is centred at $(0,-0.2) \mathrm{m}$, and it has an exterior radius of $0.6 \mathrm{~m}$ and an inner radius of $0.3 \mathrm{~m}$. Belkebir and Tijhuis, and Litman et al. have used 64 sources and 65 receivers on a circle of radius $3 \mathrm{~m}$ centred at $(0,0)$, while the inverting domain was discretized into $30 \times 30$ cells. van den Berg et al. have taken 48 source/receiver stations, while the inverting domain was discretized into $64 \times 64$ cells. In our simulation, 36 source/receiver stations are used and uniformly distributed on the same circle, which means we have under-sampled this problem further. In our simulation, $P=Q=36$, viz., we have $36 \times 36$ measurement data for TM case and $36 \times 72$ measurement data for TE case. Same objects but of different relative permittivity have been considered. The conductivity is fixed at $10 \mathrm{mS} / \mathrm{m}$, so we have the same attenuation, while the relative permittivity attains values $-2.0,2.5,3.0$, and 3.5, respectively. The operating frequency is $300 \mathrm{MHz}$, therefore, the corresponding values of the contrast are $\chi=1.0-0.6 \mathrm{i}$, $\chi=1.5-0.6 \mathrm{i}, \chi=2.0-0.6 \mathrm{i}$ and $\chi=2.5-0.6 \mathrm{i}$, respectively. The original "Austria" profile is given in Fig. 2, in which the green dots represent the 36 source/receiver stations.

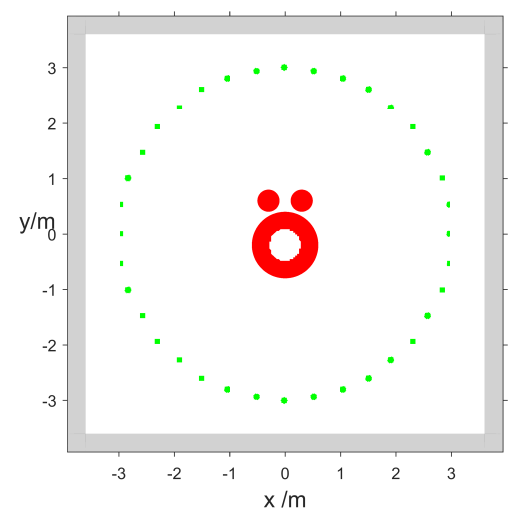

Fig. 2. The original "Austria" profile contained in a region of $[-4,4] \times[-4,4]$ $\mathrm{m}^{2}$. The green dots represent the 36 source/receiver stations. The boundaries of the four sides are terminated with PMLs. The two $z$-normal boundaries are subject to PBC.

The forward EM scattering problem is solved by a MATLAB-based 3D FDFD package "MaxwellFDFD" [33]. The $x$ - and $y$-normal boundaries are covered by perfect matching layers (PML) to simulate the anechoic chamber environment (see the gray layers of Fig. 2 at the boundaries of the test domain), while the two $z$-normal boundaries are subject to periodic boundary conditions (PBC) to simulate the
2D configuration. Line sources parallel to the $z$-axis are used to generate TM-polarized and TE-polarized incident wave. Non-uniform meshes are used to generate the scattered data, which means the testing domain is discretized with different mesh sizes determined by the distribution of the permittivity, viz., coarse meshes for low permittivity and fine meshes for high permittivity. The accuracy of the FDFD scheme is ensured by the following criterion [34]

$$
\Delta \leq \frac{\lambda_{0}}{15 \sqrt{\varepsilon_{r}}},
$$

where, $\lambda_{0}$ is the wavelength in free space. Non-uniform meshes greatly reduce the computational burden for solving the forward scattering problem. In contrast, uniform meshes are used to invert the scattered data, since we do not know the distribution of the permittivity beforehand. To guarantee the inverting accuracy, the following condition is satisfied

$$
\Delta \leq \frac{\lambda_{0}}{15 \sqrt{\max \left\{\varepsilon_{r}\right\}}} .
$$

The scattered field is obtained by subtracting the incident field from the total field.

\section{B. Condition number of the measurement matrix $\mathbf{\Phi}$}

In the following simulations, the inversion domain is restricted to the region $[-1.5,1.5] \times[-1.5,1.5] \mathrm{m}^{2}$. The dimension of the mesh grid is $30 \times 30 \mathrm{~mm}^{2}$. Thus we have specifically in this simulation, $\Phi \in \mathbb{C}^{36 \times 10000}$ for TM polarization, and $\Phi \in \mathbb{C}^{72 \times 20000}$ for $\mathrm{TE}$ polarization. With corresponding condition numbers of $\boldsymbol{\Phi}$ for TM polarization yields $\kappa(\boldsymbol{\Phi})_{T M}=5.19 \times 10^{2}$ and $\kappa(\boldsymbol{\Phi})_{T E}=8.25 \times 10^{7}$ for TE polarization, respectively. As one can see the condition number of the matrix $\boldsymbol{\Phi}$ is large for both TM and TE polarization, indicating that an error in the contrast sources $\boldsymbol{j}_{p}$ may cause an increased error in the measurement data $f_{p}$. In addition, compared to TM polarization, TE polarization is more illconditioned because $\kappa(\boldsymbol{\Phi})_{T E}$ is much larger than $\kappa(\boldsymbol{\Phi})_{T M}$, due to the different formulation of the scattering equations. This implies that the introduction of the cross-correlated cost functional has higher influence on a TE case than a TM case, which is demonstrated by the following simulation results. It is worth noting that in the formulation of TE scattering problems, the operators involved have the same form, but one spatial dimension lower, compared to full 3D scattering problems. Hence, the performance gain with CCCSI in future 3D inversion problems can be compared to the performance gain in the TE case.

\section{Noise-free data}

For fair comparison, in the following simulations, the contrast sources are initialized by Eq. (21) and the contrast is initialized by Eq. (22) for all the three algorithms. Since the background of this benchmark problem is free space, viz., $\Re\{\chi\} \geq 0$ and $\Im\{\chi\} \leq 0$, we exploit this a priori information by simply enforcing the negative real part and the positive imaginary part of the contrast to zero after each update of the contrast [26]. Let us first investigate the inversion performance 

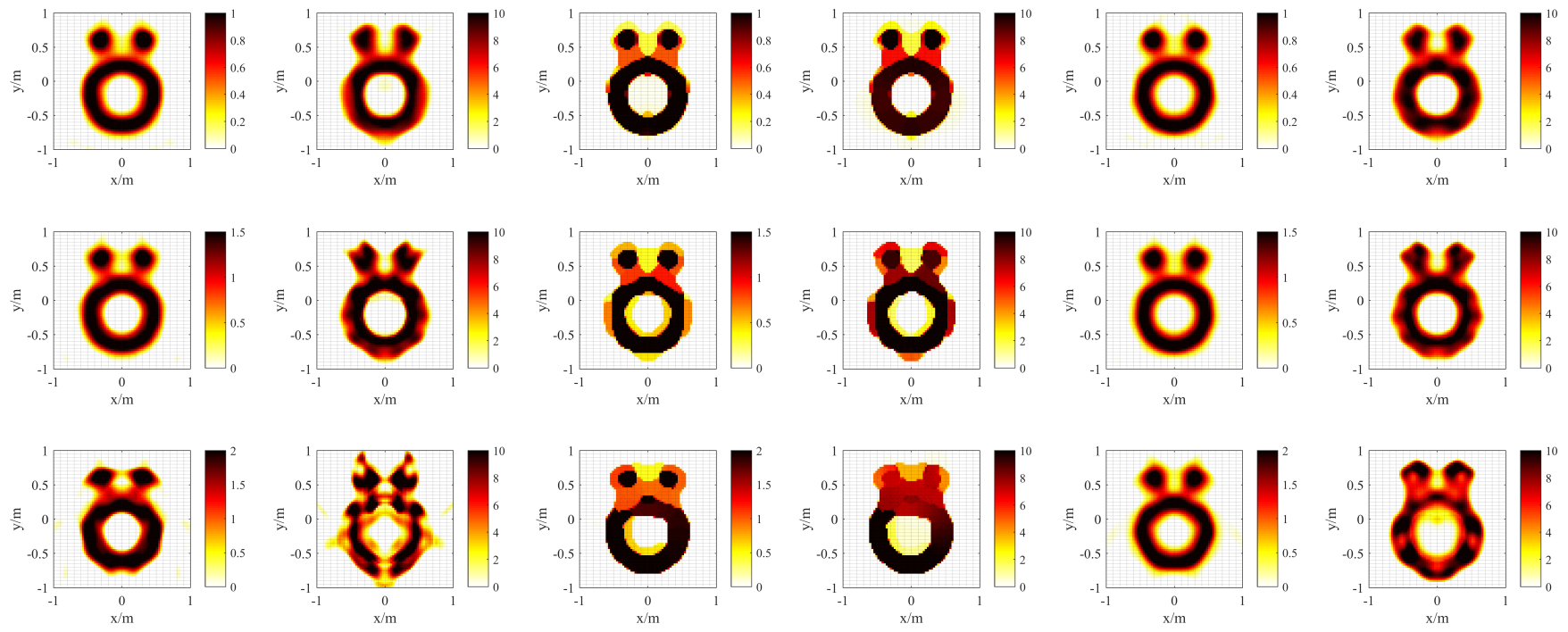

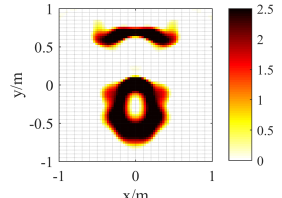

CSI, $\Delta \varepsilon_{r}$

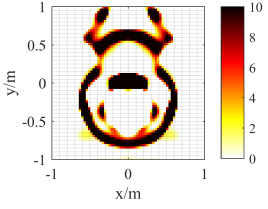

CSI, $\Delta \sigma(\mathrm{mS} / \mathrm{m})$

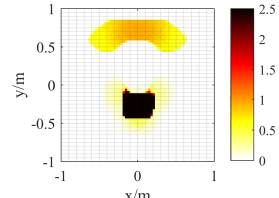

MR-CSI, $\Delta \varepsilon_{r}$

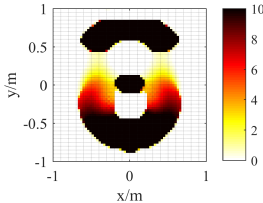

MR-CSI, $\Delta \sigma(\mathrm{mS} / \mathrm{m})$

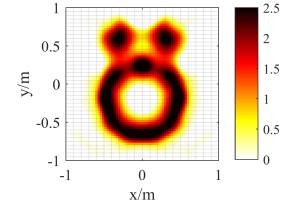

CCCSI, $\Delta \varepsilon_{r}$

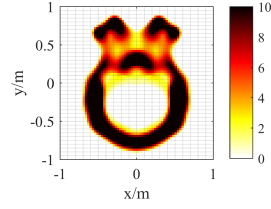

CCCSI, $\Delta \sigma(\mathrm{mS} / \mathrm{m})$

Fig. 3. The relative permittivity and conductivity of the contrast obtained by classical CSI, MR-CSI, and CCCSI, respectively, after 2048 iterations. The noise-free TM-polarized data at $300 \mathrm{MHz}$ is processed. From top to bottom: $\chi=1.0-0.6 \mathrm{i}, 1.5-0.6 \mathrm{i}, 2.0-0.6 \mathrm{i}$, and $2.5-0.6 \mathrm{i}$.

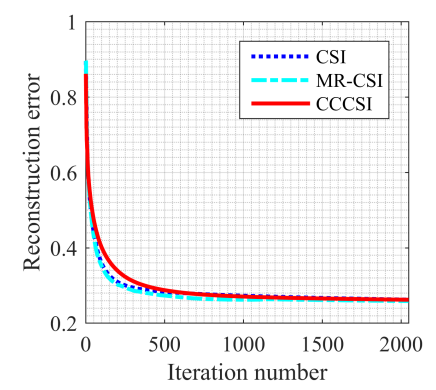

(a)

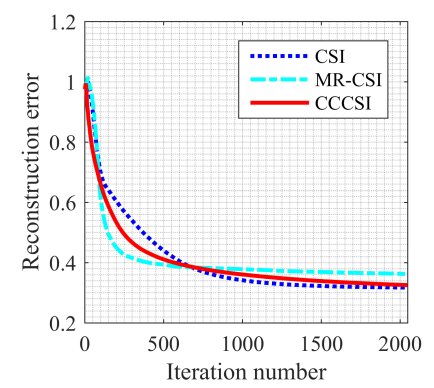

(c)

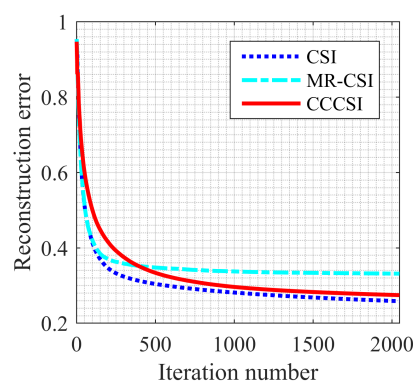

(b)

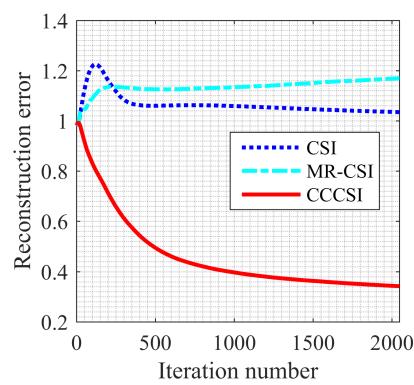

(d)

Fig. 4. The reconstruction error curves of classical CSI, MR-CSI, and CCCSI, in terms of the iteration number in the noise-free TM case. The operating frequency is $300 \mathrm{MHz}$. (a) $\chi=1.0-0.6 \mathrm{i}$; (b) $\chi=1.5-0.6 \mathrm{i}$; (c) $\chi=$ $2.0-0.6 \mathrm{i}$; (d) $\chi=2.5-0.6 \mathrm{i}$.

to the noise-free data. Both the TM-polarized data and the TEpolarized data are processed by classical CSI, MR-CSI, and CCCSI, respectively. The relative permittivity and conductivity

of the reconstructed contrast after 2048 iterations are shown in Fig. 3 for the TM case and Fig. 5 for the TE case. From Fig. 3 and Fig. 5 we see that MR-CSI generates blocky images because of the introduction of the total variation constraint, while classical CSI and CCCSI have obvious variation in the reconstructed images. As we can see CCCSI show better robustness by noting from Fig. 3 that the images of the contrast $\chi=2.0-0.6 \mathrm{i}$ obtained by classical CSI and MR-CSI show more distortion than those of CCCSI, and that classical CSI and MR-CSI fail to reconstruct the contrast of $\chi=2.5-0.6 \mathrm{i}$ using the TM-polarized data. We can also see From Fig. 5 that MR-CSI fail to reconstruct the contrast $\chi=2.0-0.6 \mathrm{i}$ using the noise-free TE-polarized data. In addition, we see that the interior hollow tube is better reconstructed by CCCSI in the TE case, and the two smaller tubes are better distinguished by CCCSI compared to classical CSI and MR-CSI.

To quantitatively investigate the reconstruction accuracy, the reconstruction error of the three inversion methods is defined in the following as

$$
\text { err }=\frac{\|\chi-\hat{\chi}\|^{2}}{\|\chi\|^{2}}
$$

Fig. 4 and Fig. 6 give the comparison of the reconstruction error curves in terms of the iteration number of the three methods in the TM case and the TE case, respectively. As we can see quantitatively that, in the TM case, the three methods reach the same reconstruction errors in reconstructing the contrasts $\chi=1.0-0.6 \mathrm{i}, 1.5-0.6 \mathrm{i}$, and $2.0-0.6 \mathrm{i}$. However, the reconstruction errors of classical CSI and MR-CSI do not decrease in reconstructing the contrast $\chi=2.5-0.6 \mathrm{i}$. 

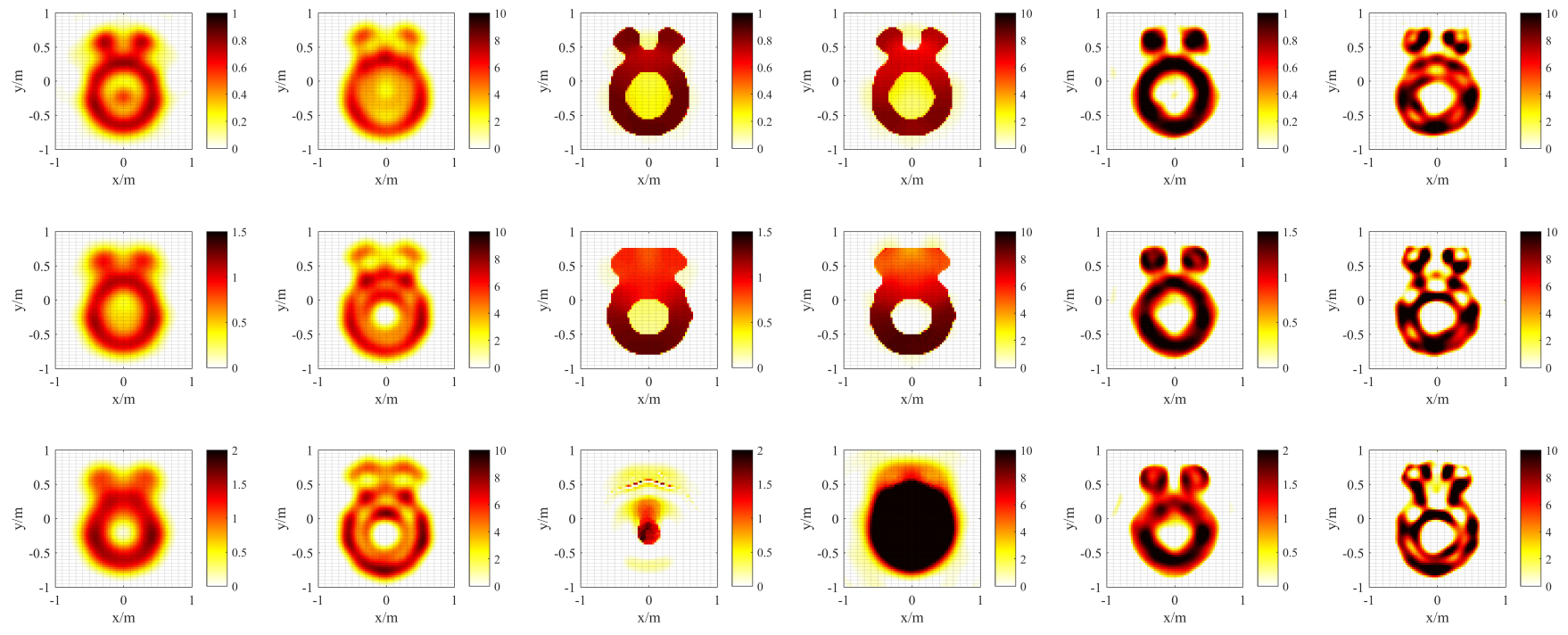

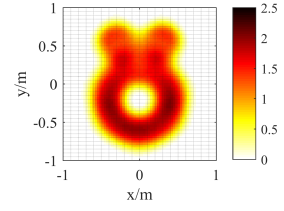

CSI, $\varepsilon$

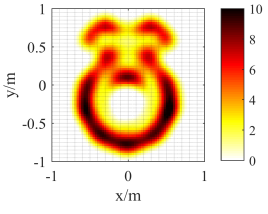

CSI, $\Delta \sigma(\mathrm{mS} / \mathrm{m})$

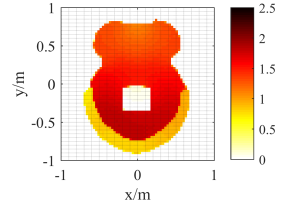

MR-CSI, $\varepsilon$

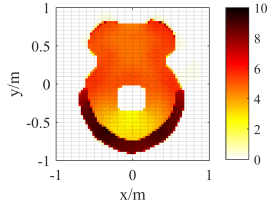

MR-CSI, $\Delta \sigma(\mathrm{mS} / \mathrm{m})$

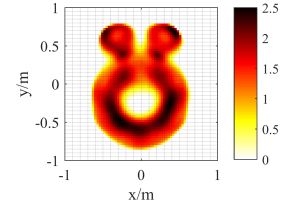

CCCSI, $\varepsilon$

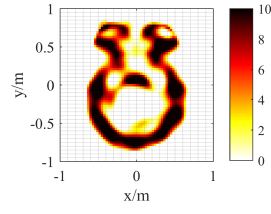

CCCSI, $\Delta \sigma(\mathrm{mS} / \mathrm{m})$

Fig. 5. The relative permittivity and conductivity of the contrast obtained by classical CSI, MR-CSI, and CCCSI, respectively, after 2048 iterations. The noise-free TE-polarized data at $300 \mathrm{MHz}$ is processed. From top to bottom: $\chi=1.0-0.6 \mathrm{i}, 1.5-0.6 \mathrm{i}, 2.0-0.6 \mathrm{i}$, and $2.5-0.6 \mathrm{i}$.

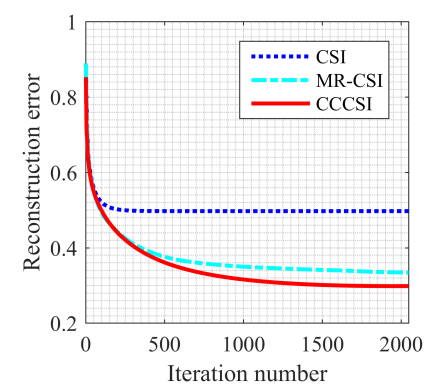

(a)

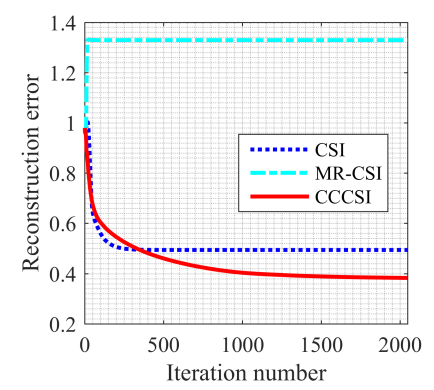

(c)

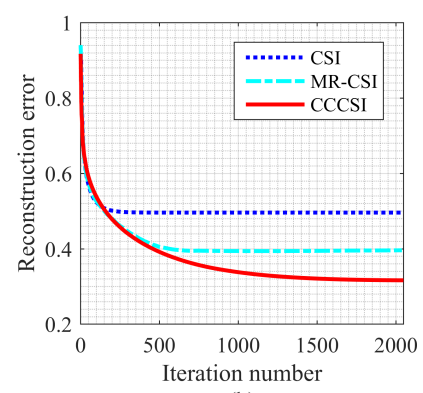

(b)

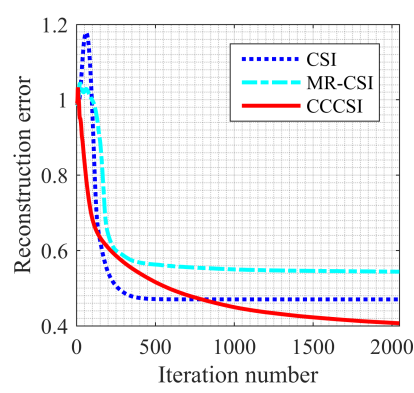

(d)

Fig. 6. The reconstruction error curves of classical CSI, MR-CSI, and CCCSI, in terms of the iteration number in the noise-free TE case. The operating frequency is $300 \mathrm{MHz}$. (a) $\chi=1.0-0.6 \mathrm{i}$; (b) $\chi=1.5-0.6 \mathrm{i}$; (c) $\chi=$ $2.0-0.6 \mathrm{i}$; (d) $\chi=2.5-0.6 \mathrm{i}$.

In the contrast, the decreasing tendency of the reconstruction error curve of CCCSI is not obviously affected by increasing the value of the contrast. In the TE case, we can see from

Fig. 6 that CCCSI can achieve lower reconstruction errors than classical CSI and MR-CSI, indicating the higher inversion accuracy of CCCSI compared to classical CSI and MRCSI. The reconstruction error of MR-CSI in reconstructing the contrast $\chi=2.0-0.6 \mathrm{i}$ does not decrease, and the reconstruction error curve of classical CSI in reconstructing the contrast $\chi=2.5-0.6 \mathrm{i}$ shows non-monotonicity. This demonstrates the poor robustness of both classical CSI and MR-CSI, and the better robustness of CCCSI.

It is worth noting that CCCSI shows higher inversion accuracy than classical CSI and MR-CSI in the TE case, but similar inversion accuracy with classical CSI and MR-CSI in TM case. Recalling the previous subsection, we know that the matrix $\boldsymbol{\Phi}$ of TE polarization has a much larger condition number than that of TM polarization. Therefore, compared to the TM case, same level of cross-correlated error presented in the measurement domain in TE case corresponds to a smaller reconstruction error in the field domain.

In addition, as we can see from the simulation results, MR-CSI shows not only poor robustness, but also unstable performance with respect to inversion accuracy compared to classical CSI and CCCSI. As is well known, total variation regularization was originally proposed for noise removal in the digital image processing [35]. Obviously, the feasibility condition for applying the total variation regularization is that this noisy image is suitable for processing. However, this is apparently not the case in CSI, because the image of the contrast in CSI is optimized iteratively. In the design of MRCSI, total variation constraint is very likely to be applied to 

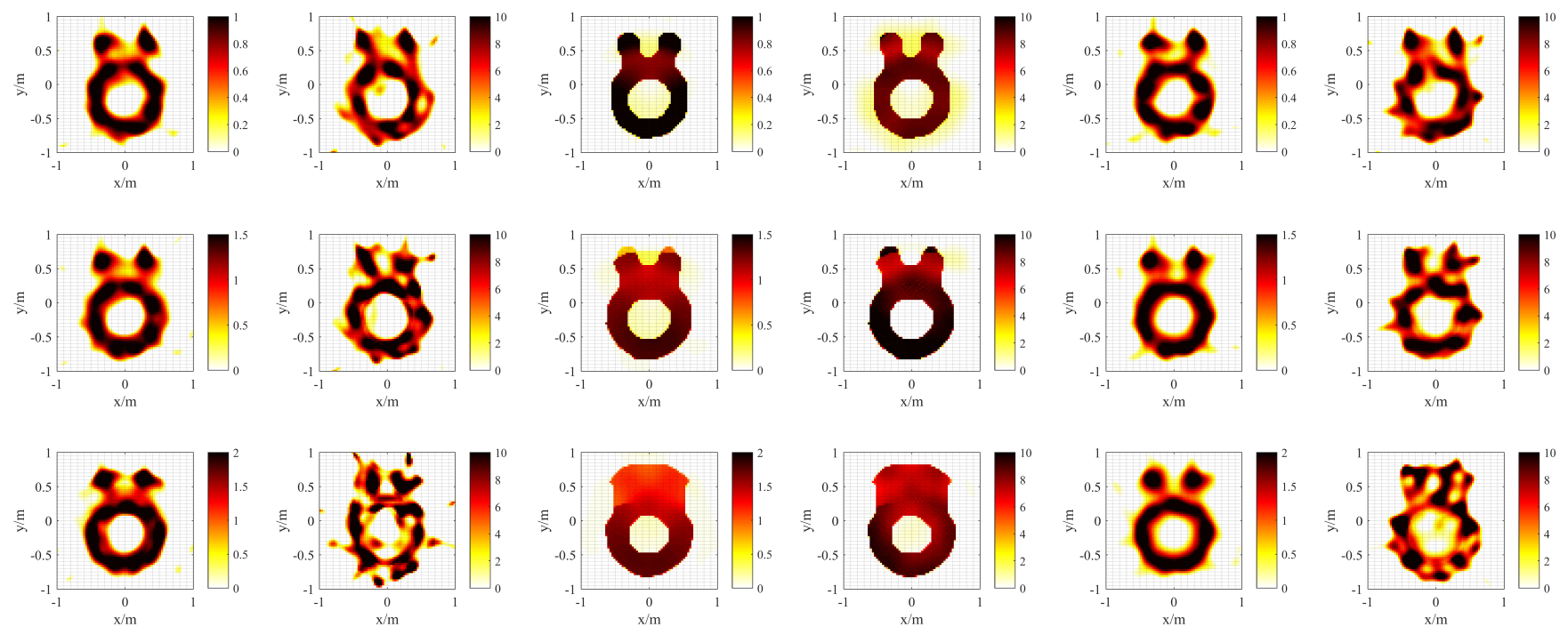

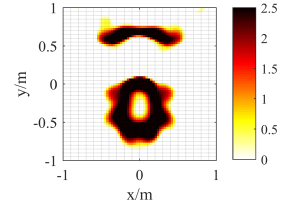

CSI, $\varepsilon$

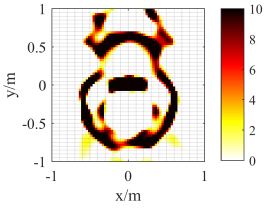

CSI, $\Delta \sigma(\mathrm{mS} / \mathrm{m})$

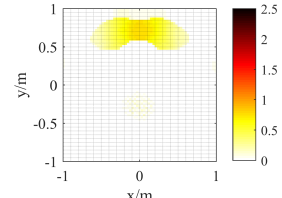

MR-CSI, $\varepsilon$

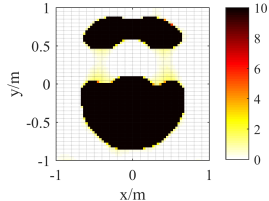

MR-CSI, $\Delta \sigma(\mathrm{mS} / \mathrm{m})$

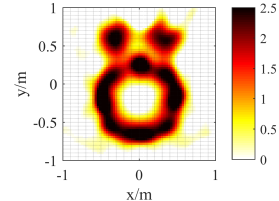

CCCSI, $\varepsilon$

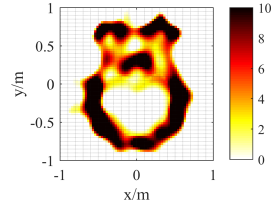

CCCSI, $\Delta \sigma(\mathrm{mS} / \mathrm{m})$

Fig. 7. The relative permittivity and conductivity of the contrast obtained by classical CSI, MR-CSI, and CCCSI, respectively, after 2048 iterations. The noise-disturbed TM-polarized data at $300 \mathrm{MHz}$ is processed. From top to bottom: $\chi=1.0-0.6 \mathrm{i}, 1.5-0.6 \mathrm{i}, 2.0-0.6 \mathrm{i}$, and $2.5-0.6 \mathrm{i}$. $10 \%$ additive random white noise is considered.

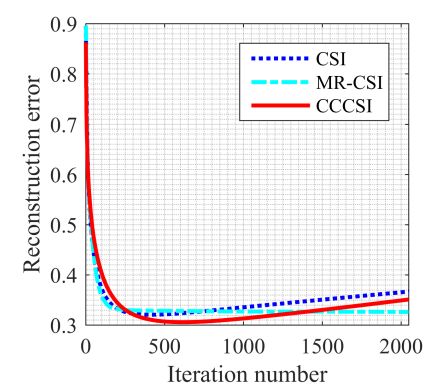

(a)

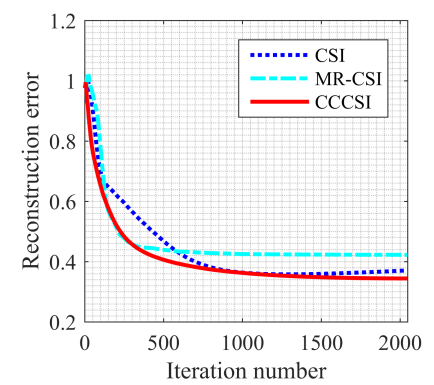

(c)

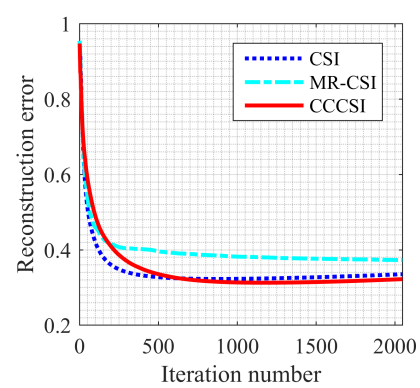

(b)

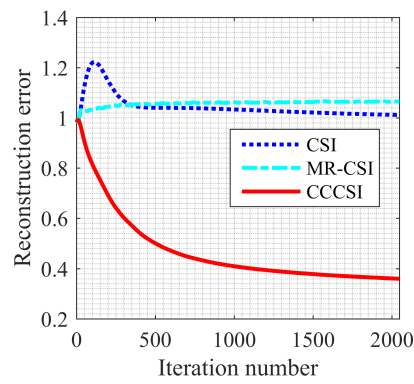

(d)

Fig. 8. The reconstruction error curves of classical CSI, MR-CSI, and CCCSI, in terms of the iteration number in the noise-disturbed TM case. The operating frequency is $300 \mathrm{MHz} .10 \%$ additive random white noise is considered. (a) $\chi=1.0-0.6 \mathrm{i}$; (b) $\chi=1.5-0.6 \mathrm{i}$; (c) $\chi=2.0-0.6 \mathrm{i}$; (d) $\chi=2.5-0.6 \mathrm{i}$.

a seriously distorted image of the contrast in the beginning, and thus may mislead and degrade the optimization process.

Therefore, benefits can be possibly obtained from MR-CSI only if the contrast can be reliably reconstructed with CSI. Namely, the benefits from MR-CSI are not guaranteed. This perfectly explains the instable performance of MR-CSI shown in Fig. 4 and Fig. 6.

\section{Noise-disturbed data}

In real applications, the measurement data are very likely to be disturbed by noises. Apart from that, there is always error in modeling the incident fields. In this subsection, we investigate the inversion performance of the three methods to noise-disturbed data while the incident fields are assumed to be exactly known. Random white noise is added to the measurement data following the same procedure used in [32],

$$
\boldsymbol{f}_{p, \text { noise }}=\boldsymbol{f}_{p}+\zeta \times \max _{m}\left\{\left|f_{p, m}\right|\right\}\left(\boldsymbol{n}_{1}+\mathrm{i} \boldsymbol{n}_{2}\right)
$$

with $p=1,2, \cdots, P, m=1,2, \cdots, M$. Here, $\boldsymbol{n}_{1}$ and $\boldsymbol{n}_{2}$ are two random numbers varying from -1 up to $1, \zeta=10 \%$ is the amount of noise, and $\max _{m}\left\{\left|f_{p, m}\right|\right\}$ gives the largest value among the amplitudes of the $M$ measurement data, which means the noise is scaled by the largest amplitude of the measurement data. $M=36$ in the TM case and $M=72$ in the TE case.

Fig. 7 and Fig. 9 show the inverted results after 2048 iterations by the three methods using the TM-polarized data and the TE-polarized data, respectively. In comparison to Fig. 3 and Fig. 5, we can see obvious distortion in the reconstructed images because of the disturbance by the additive random 

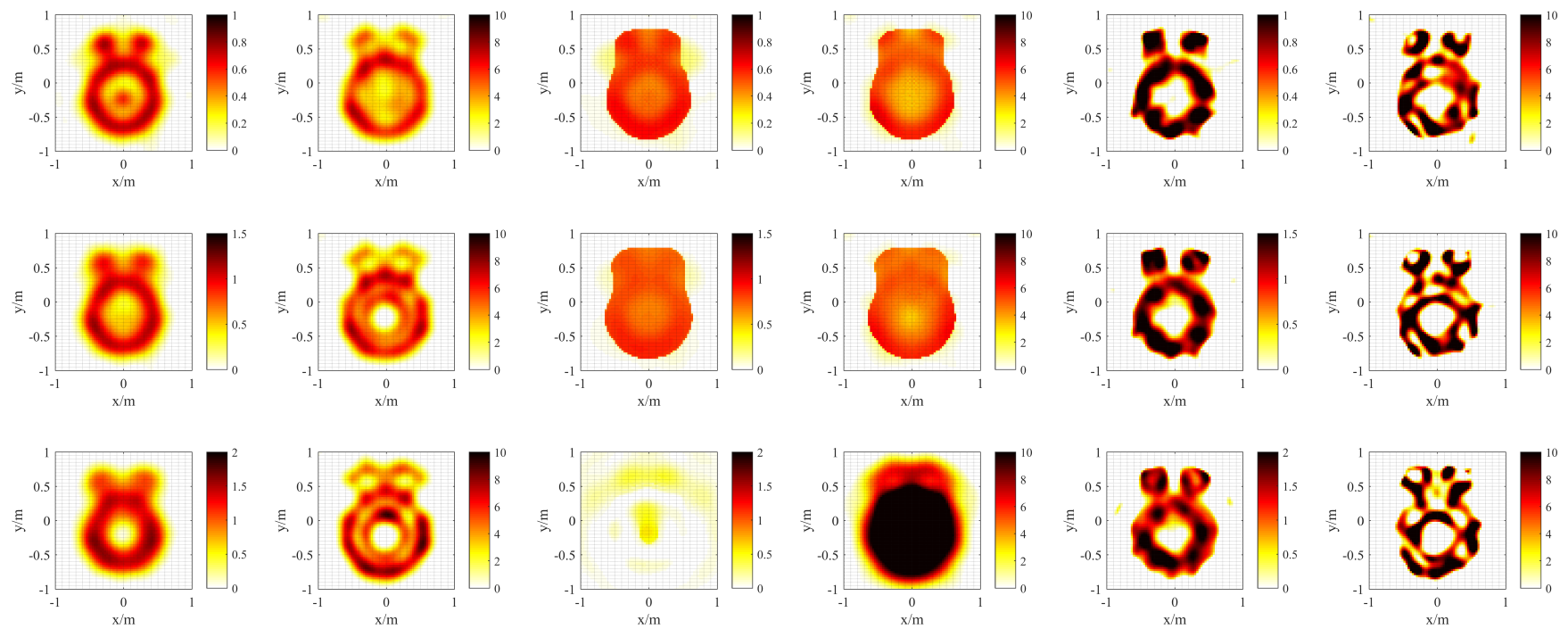

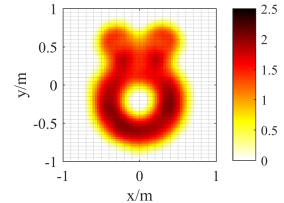

CSI, $\varepsilon$

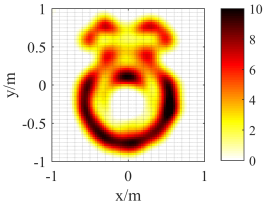

CSI, $\Delta \sigma(\mathrm{mS} / \mathrm{m})$

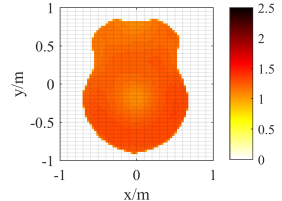

MR-CSI, $\varepsilon$

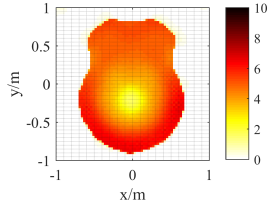

MR-CSI, $\Delta \sigma(\mathrm{mS} / \mathrm{m})$

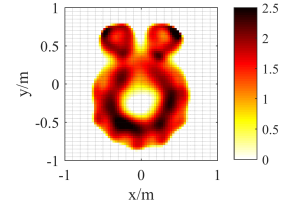

CCCSI, $\varepsilon$

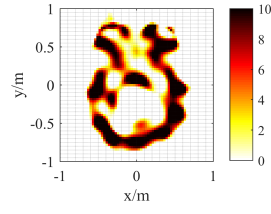

CCCSI, $\Delta \sigma(\mathrm{mS} / \mathrm{m})$

Fig. 9. The relative permittivity and conductivity of the contrast obtained by classical CSI, MR-CSI, and CCCSI, respectively, after 2048 iterations. The noise-disturbed TE-polarized data at $300 \mathrm{MHz}$ is processed. From top to bottom: $\chi=1.0-0.6 \mathrm{i}, 1.5-0.6 \mathrm{i}, 2.0-0.6 \mathrm{i}$, and $2.5-0.6 \mathrm{i}$. $10 \%$ additive random white noise is considered.

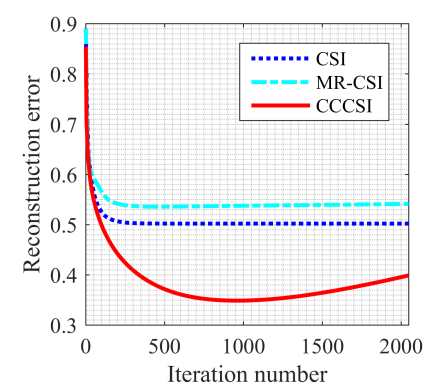

(a)

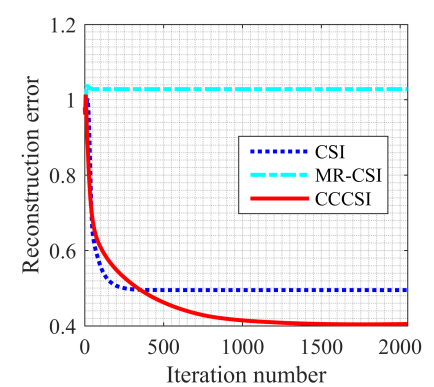

(c)

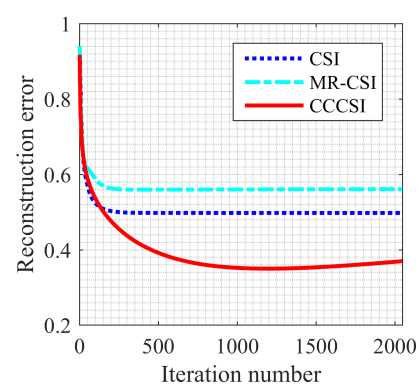

(b)

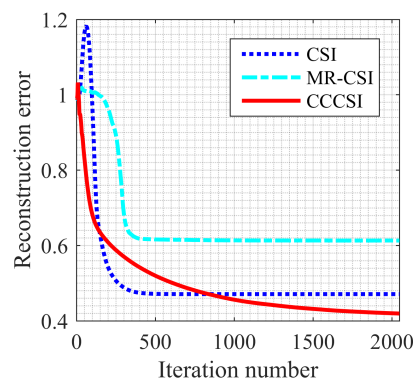

(d)

Fig. 10. The reconstruction error curves of classical CSI, MR-CSI, and CCCSI, in terms of the iteration number in the noise-disturbed TE case. The operating frequency is $300 \mathrm{MHz} .10 \%$ additive random white noise is considered. (a) $\chi=1.0-0.6 \mathrm{i}$; (b) $\chi=1.5-0.6 \mathrm{i}$; (c) $\chi=2.0-0.6 \mathrm{i}$; (d) $\chi=2.5-0.6 \mathrm{i}$.

noise. What's worse, we see from Fig. 9 that the hollow

tube is not distinguishable any more in the images obtained by MR-CSI using the noise-disturbed TE-polarized data. As mentioned previously in Subsection IV-C, since the hollow tube cannot be well recognized by CSI (see the images obtained by CSI in Fig. 9), we lose the basic feasibility condition for applying the total variation constraint and therefore the hollow tube is reconstructed by MR-CSI to a solid one. In the contrast, CCCSI is still capable to distinguish the hollow tube even though the measurement data has been disturbed by $10 \%$ additive random noise.

The reconstruction error curves of classical CSI, MR-CSI and CCCSI in the noise-disturbed cases are given in Fig. 8 for the TM case and Fig. 10 for the TE case. As we can see, the error curves of classical CSI and MR-CSI show instability as the contrast goes higher, while the error curves of CCCSI are always monotonously decreasing, indicating again the better robustness of CCCSI. We can also see from Fig. 10 that CCCSI has lower reconstruction error than classical CSI and MR-CSI, which is consistent with the simulation results in the noise-free cases.

What's more, from Fig. 8(a) we see that the reconstruction errors of classical CSI and CCCSI turn out to increase as the iteration goes on. Same phenomenon also occurs in Fig. 10(a,b) for CCCSI. By comparison to Fig. 4(a) and Fig. 6(a,b) in the noise-free case, this phenomenon can been easily explained by the introduction of the additive random noise. Therefore, in real applications, a good termination condition is critical for preventing the methods from over-fitting the noise and for saving computation time. 


\section{E. Computational performance}

As mentioned previously, CCCSI can be implemented without significantly increasing the computational complexity. To demonstrate this point, we run the MATLAB codes on a desktop with one Intel(R) Core(TM) i5-3470 CPU @ 3.20 $\mathrm{GHz}$, and we did not use parallel computing. The computation times of classical CSI, MR-CSI, and CCCSI, running for 2048 iterations are given in Table I.

TABLE I

THE COMPUTATIONAL TIMES

\begin{tabular}{|c|c|c|c|}
\hline Time $/ \mathrm{s}$ & CSI & MR-CSI & CCCSI \\
\hline Polarization & & & \\
\hline TM & 1361.2 & 1419.7 & 1429.6 \\
\hline TE & 2931.9 & 3040.9 & 3146.2 \\
\hline
\end{tabular}

As we can see CSI is the most efficient, MR-CSI is in the middle, and CCCSI runs slightly longer. If we define the increment percentage of the running times of CCCSI as

$$
\frac{T_{\mathrm{CCCSI}}-T_{\mathrm{CSI}}}{T_{\mathrm{CSI}}} \times 100 \%
$$

we have that the running time of CCCSI is slightly longer than that of classical CSI by around 5.0\% in the TM case and $7.3 \%$ in the TE case.

\section{CONClusion}

In this paper, a cross-correlated contrast source inversion (CCCSI) method is proposed by modifying the cost functional of the CSI method to interrelate the state error and the data error. The proposed algorithm is tested with a $2 \mathrm{D}$ benchmark problem which has also been tested by Belkebir and Tijhuis [30], Litman et al. [31], and Berg et al. [26], [32]. Simulation results with both TM-polarized wave and TE-polarized wave show that CCCSI outperforms classical CSI and MR-CSI with respect to robustness and inversion accuracy, especially in the TE case. Which shows to be promising for the robustness and inversion accuracy in full 3D inversion problems. We have also shown that CCCSI can be implemented without significantly increasing the computational burden. As the Maxwell equations are formulated within a 3D finite difference frequency domain (FDFD) scheme, it is straightforward to extend the proposed inversion scheme to future 3D inverse scattering problems. Numerical results of 3D scattering objects, including the application of the proposed method to experimental data will be published in future work.

\section{APPENDIX A}

DERIVATION OF THE STEP SIZE $\alpha_{p, n}$

First, let us rewrite the cost function $C_{\mathrm{CCCSI}}^{j}\left(\chi_{n-1}, \boldsymbol{j}_{p, n-1}+\right.$ $\left.\alpha_{p} \boldsymbol{\nu}_{p, n}\right)$ as follows

$$
\begin{aligned}
& C_{\mathrm{CCCSI}}^{\boldsymbol{j}}\left(\boldsymbol{\chi}_{n-1}, \boldsymbol{j}_{p, n-1}+\alpha_{p} \boldsymbol{\nu}_{p, n}\right)= \\
& \eta^{\mathcal{S}} \sum_{p=1}^{P}\left\|\boldsymbol{\rho}_{p, n-1}-\alpha_{p} \boldsymbol{\Phi} \boldsymbol{\nu}_{p, n}\right\|^{2}+ \\
& \eta_{n-1}^{\mathcal{D}} \sum_{p=1}^{P}\left\|\boldsymbol{\gamma}_{p, n-1}+\alpha_{p}\left(\boldsymbol{\chi}_{n-1} \mathcal{M}_{\mathcal{D}} \boldsymbol{A}^{-1}-\boldsymbol{I}\right) \boldsymbol{\nu}_{p, n}\right\|^{2}+ \\
& \eta^{\mathcal{S}} \sum_{p=1}^{P}\left\|\boldsymbol{\xi}_{p, n-1}-\alpha_{p} \boldsymbol{\Phi} \boldsymbol{\chi}_{n-1} \mathcal{M}_{\mathcal{D}} \boldsymbol{A}^{-1} \boldsymbol{\nu}_{p, n}\right\|^{2}
\end{aligned}
$$

Obviously, it can be further simplified in the form of

$$
\begin{aligned}
& C_{\mathrm{CCCSI}}^{\boldsymbol{j}}\left(\boldsymbol{\chi}_{n-1}, \boldsymbol{j}_{p, n-1}+\alpha_{p} \boldsymbol{\nu}_{p, n}\right)= \\
& a_{p, 2} \alpha_{p, n}^{2}+a_{p, 1} \alpha_{p, n}+a_{p, 0}+b_{p, 2} \alpha_{p}^{2}+b_{p, 1} \alpha_{p}+b_{p, 0}+ \\
& c_{p, 2} \alpha_{p, n}^{2}+c_{p, 1} \alpha_{p}+c_{p, 0} .
\end{aligned}
$$

Therefore, we have

$$
\begin{aligned}
\alpha_{p, n} & =\max _{\alpha_{p}} C_{\mathrm{CCCSI}}^{j}\left(\boldsymbol{\chi}_{n-1}, \boldsymbol{j}_{p, n-1}+\alpha_{p} \boldsymbol{\nu}_{p, n}\right) \\
& =-\frac{1}{2} \frac{a_{p, 1}+b_{p, 1}+c_{p, 1}}{a_{p, 2}+b_{p, 2}+c_{p, 2}} .
\end{aligned}
$$

Note that

$$
\begin{aligned}
& a_{p, 2}=\eta^{\mathcal{S}}\left\|\boldsymbol{\Phi} \boldsymbol{\nu}_{p, n}\right\|_{\mathcal{S}}^{2}, \\
& a_{p, 1}=-2 \eta^{\mathcal{S}}\left\{\left\{\boldsymbol{\nu}_{p, n}^{H} \boldsymbol{\Phi}^{H} \boldsymbol{\rho}_{p, n-1}\right\},\right.
\end{aligned}
$$

$$
\begin{aligned}
& b_{p, 2}=\eta_{n-1}^{\mathcal{D}}\left\|\boldsymbol{\nu}_{p, n}-\boldsymbol{\chi} \mathcal{M}_{\mathcal{D}} \boldsymbol{A}^{-1} \boldsymbol{\nu}_{p, n}\right\|_{\mathcal{D}}^{2}, \\
& b_{p, 1}=2 \eta_{n-1}^{\mathcal{D}} \Re\left\{\boldsymbol{\nu}_{p, n}^{H}\left(\boldsymbol{\chi} \mathcal{M}_{\mathcal{D}} \boldsymbol{A}^{-1}-\boldsymbol{I}\right)^{H} \boldsymbol{\gamma}_{p, n-1}\right\}, \\
& c_{p, 2}=\eta^{\mathcal{S}}\left\|\boldsymbol{\Phi} \boldsymbol{\chi} \mathcal{M}_{\mathcal{D}} \boldsymbol{A}^{-1} \boldsymbol{\nu}_{p, n}\right\|_{\mathcal{S}}^{2} \\
& c_{p, 1}=-2 \eta^{\mathcal{S}} \Re\left\{\boldsymbol{\nu}_{p, n}^{H}\left(\boldsymbol{\Phi} \boldsymbol{\chi} \mathcal{M}_{\mathcal{D}} \boldsymbol{A}^{-1}\right)^{H} \boldsymbol{\xi}_{p, n-1}\right\},
\end{aligned}
$$

and

$$
\begin{aligned}
\boldsymbol{g}_{p, n}= & -2 \eta^{\mathcal{S}} \boldsymbol{\Phi}^{H} \boldsymbol{\rho}_{p, n-1}+ \\
& 2 \eta_{n-1}^{\mathcal{D}}\left(\boldsymbol{\chi} \mathcal{M}_{\mathcal{D}} \boldsymbol{A}^{-1}-\boldsymbol{I}\right)^{H} \gamma_{p, n-1}- \\
& 2 \eta^{\mathcal{S}}\left(\boldsymbol{\Phi} \boldsymbol{\chi} \mathcal{M}_{\mathcal{D}} \boldsymbol{A}^{-1}\right)^{H} \boldsymbol{\xi}_{p, n-1},
\end{aligned}
$$

it is easy to obtain that

$$
\alpha_{p, n}=-\frac{\Re\left\{\left\langle\boldsymbol{g}_{p, n}, \boldsymbol{\nu}_{p, n}\right\rangle_{\mathcal{D}}\right\}}{2\left(a_{p, 2}+b_{p, 2}+c_{p, 2}\right)} .
$$

where, $a_{p, 2}, b_{p, 2}$, and $c_{p, 2}$ are given by Eq. (32a), Eq. (33a), and Eq. (34a), respectively. 


\section{REFERENCES}

[1] A. Klotzsche, J. van der Kruk, A. Mozaffari, N. Gueting, and H. Vereecken, "Crosshole GPR full-waveform inversion and waveguide amplitude analysis: Recent developments and new challenges," in 2015 8th International Workshop on Advanced Ground Penetrating Radar (IWAGPR). IEEE, 2015, pp. 1-6.

[2] W. Hu, A. Abubakar, and T. M. Habashy, "Simultaneous multifrequency inversion of full-waveform seismic data," Geophysics, vol. 74, no. 2, pp. R1-R14, 2009.

[3] A. Rosenthal, V. Ntziachristos, and D. Razansky, "Acoustic inversion in optoacoustic tomography: A review," Current medical imaging reviews, vol. 9, no. 4, pp. 318-336, 2013.

[4] C. Gilmore, A. Abubakar, W. Hu, T. M. Habashy, and P. M. Van Den Berg, "Microwave biomedical data inversion using the finitedifference contrast source inversion method," IEEE Transactions on Antennas and Propagation, vol. 57, no. 5, pp. 1528-1538, 2009.

[5] M. Serhir, P. Besnier, and M. Drissi, "An accurate equivalent behavioral model of antenna radiation using a mode-matching technique based on spherical near field measurements," IEEE Transactions on Antennas and Propagation, vol. 56, no. 1, pp. 48-57, Jan 2008.

[6] M. Serhir, J.-M. Geffrin, A. Litman, and P. Besnier, "Aperture antenna modeling by a finite number of elemental dipoles from spherical field measurements," IEEE Transactions on Antennas and Propagation, vol. 58, no. 4, pp. 1260-1268, April 2010.

[7] S. Nounouh, C. Eyraud, A. Litman, and H. Tortel, "Quantitative imaging with incident field modeling from multistatic measurements on line segments," Antennas and Wireless Propagation Letters, IEEE, vol. 14, pp. 253-256, 2015.

[8] D. Colton, M. Piana, and R. Potthast, "A simple method using morozov's discrepancy principle for solving inverse scattering problems," Inverse Problems, vol. 13, no. 6, pp. 1477-1493, 1997.

[9] D. Colton and A. Kirsch, "A simple method for solving inverse scattering problems in the resonance region," Inverse problems, vol. 12, no. 4, pp. 383-393, 1996.

[10] P. M. Van Den Berg and R. E. Kleinman, "A contrast source inversion method," Inverse problems, vol. 13, no. 6, pp. 1607-1620, 1997.

[11] B. Kooij, M. Lambert, and D. Lesselier, "Nonlinear inversion of a buried object in transverse electric scattering," Radio Science, vol. 34, no. 6, pp. 1361-1371, 1999

[12] P. M. van den Berg, A. Van Broekhoven, and A. Abubakar, "Extended contrast source inversion," Inverse Problems, vol. 15, no. 5, pp. 1325$1344,1999$.

[13] T. Isernia, L. Crocco, and M. D'Urso, "New tools and series for forward and inverse scattering problems in lossy media," Geoscience and Remote Sensing Letters, IEEE, vol. 1, no. 4, pp. 327-331, 2004.

[14] L. Crocco, M. D'Urso, and T. Isernia, "The contrast source-extended Born model for 2D subsurface scattering problems," Progress In Electromagnetics Research B, vol. 17, pp. 343-359, 2009.

[15] A. Abubakar, W. Hu, P. Van Den Berg, and T. Habashy, "A finite-difference contrast source inversion method," Inverse Problems, vol. 24, no. 6, p. 065004, 2008. [Online]. Available: http://stacks.iop. org $/ 0266-5611 / 24 / \mathrm{i}=6 / \mathrm{a}=065004$

[16] A. Abubakar, G. Pan, M. Li, L. Zhang, T. Habashy, and P. van den Berg, "Three-dimensional seismic full-waveform inversion using the finitedifference contrast source inversion method," Geophysical Prospecting, vol. 59, no. 5, pp. 874-888, 2011.

[17] A. Zakaria, C. Gilmore, and J. LoVetri, "Finite-element contrast source inversion method for microwave imaging," Inverse Problems, vol. 26, no. 11, p. $115010,2010$.

[18] A. Zakaria and J. LoVetri, "The finite-element method contrast source inversion algorithm for 2D transverse electric vectorial problems," IEEE Transactions on Antennas and Propagation, vol. 60, no. 10, pp. 47574765, 2012.

[19] E. A. Attardo, G. Vecchi, and L. Crocco, "Contrast source extended Born inversion in noncanonical scenarios via FEM modeling," IEEE Transactions on Antennas and Propagation, vol. 62, no. 9, pp. 4674 4685, 2014.

[20] L. Crocco, I. Catapano, L. D. Donato, and T. Isernia, "The linear sampling method as a way to quantitative inverse scattering," IEEE Transactions on Antennas and Propagation, vol. 60, no. 4, pp. 1844 1853,2012

[21] K. Ito, B. Jin, and J. Zou, "A two-stage method for inverse medium scattering," Journal of Computational Physics, vol. 237, pp. 211-223, 2013.
[22] Y.-H. Kuo and J.-F. Kiang, "A recursive approach to improve the image quality in well-logging environments," Progress In Electromagnetics Research B, vol. 60, pp. 287-300, 2014.

[23] M. Eskandari, R. Safian, and M. Dehmollaian, "Three-dimensional nearfield microwave imaging using hybrid linear sampling and level set methods in a medium with compact support," IEEE Transactions on Antennas and Propagation, vol. 62, no. 10, pp. 5117-5125, Oct 2014.

[24] D. Colton and R. Kress, Inverse acoustic and electromagnetic scattering theory. Springer Science \& Business Media, 2012, vol. 93.

[25] S. Sun, B. J. Kooij, T. Jin, and A. Yarovoy, "Simultaneous TE and TM polarization inversion based on FDFD and frequency hopping scheme in ground penetrating radar," in 8th International Workshop on Advanced Ground Penetrating Radar (IWAGPR), 2015. IEEE, 2015, pp. 1-5.

[26] P. Van Den Berg and A. Abubakar, "Contrast source inversion method: state of art," Journal of Electromagnetic Waves and Applications, vol. 15, no. 11 , pp. 1503-1505, 2001.

[27] R. P. Brent, Algorithms for minimization without derivatives. Englewood Cliffs, New Jersey, 1973

[28] G. E. Forsythe, M. A. Malcolm, and C. B. Moler, Computer Methods for Mathematical Computations. Prentice-Hall, 1976.

[29] T. M. Habashy, M. L. Oristaglio, and A. T. Hoop, "Simultaneous nonlinear reconstruction of two-dimensional permittivity and conductivity;" Radio Science, vol. 29, no. 4, pp. 1101-1118, 1994.

[30] K. Belkebir and A. Tijhuis, "Using multiple frequency information in the iterative solution of a two-dimensional nonlinear inverse problem," in Proceedings Progress in Electromagnetics Research Symposium, PIERS 1996, 8 July 1996, Innsbruck, Germany. University of Innsbruck, 1996, p. 353.

[31] A. Litman, D. Lesselier, and F. Santosa, "Reconstruction of a twodimensional binary obstacle by controlled evolution of a level-set," Inverse problems, vol. 14, no. 3, pp. 685-706, 1998.

[32] P. M. van den Berg, A. Abubakar, and J. T. Fokkema, "Multiplicative regularization for contrast profile inversion," Radio Science, vol. 38, no. $2,2003$.

[33] W. Shin. (2015) MaxwellFDFD Webpage. https://github.com/wsshin/maxwellfdfd.

[34] _ "3D finite-difference frequency-domain method for plasmonics and nanophotonics," Ph.D. dissertation, Stanford University, 2013.

[35] L. I. Rudin, S. Osher, and E. Fatemi, "Nonlinear total variation based noise removal algorithms," Physica D: Nonlinear Phenomena, vol. 60, no. 1 , pp. $259-268,1992$.

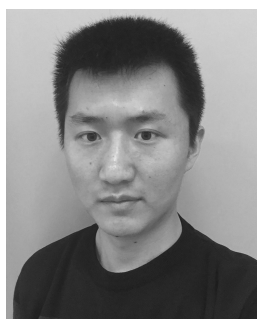

Shilong Sun received the B.S. and M.S. degrees in information and communication engineering from the National University of Defense Technology, Changsha, China, in 2011 and 2013, respectively. He is currently working toward the Ph.D. degree in the group of Microwave Sensing, Signals and Systems (MS3), Delft University of Technology, Delft, The Netherlands.

His research interests include inverse scattering problems and radar imaging. 


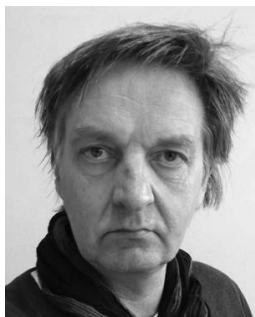

Bert Jan Kooij was born in Amersfoort, the Netherlands, on February 11, 1959. He received the B.Sc and M.Sc. degrees in electrical engineering, and the Ph.D. degree in technical sciences from Delft University of Technology, Delft, The Netherlands, in 1984, 1986, and 1994, respectively.

Since 1987, he has been a member of the scientific staff of the Electromagnetic Research Group, Delft University of Technology, where he has carried out research and taught classes in the area of electromagnetics, as well as acoustics, wave propagation, and scattering problems. During a three-month period in 1996, he was a Visiting Scientist at Ecole Supérieure dElectricité (Supelec), Gif-sur-Yvette, France. He has worked on transient wave propagation problems in the field of elastodynamics and electromagnetics. His current research interests are the computation of inverse wave-field problems employing iterative techniques based on error minimization and space time-domain wave-field modeling.

Since 2010, he has been a member of the Microwave, Sensing, Signals and Systems Group, Delft University of Technology.

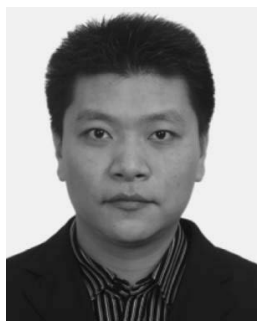

Tian Jin (S'07 M08) received the B.S., M.S., and $\mathrm{Ph} . \mathrm{D}$. degrees from the National University of Defense Technology, Changsha, China, in 2002, 2003 , and 2007, respectively, all in information and communication engineering. His Ph.D. dissertation was awarded as the National Excellent Doctoral Dissertation of China in 2009

$\mathrm{He}$ is currently a Professor with the National University of Defense Technology. He has authored/coauthored over 100 peer-reviewed papers on international journals and conferences. He has been a Visiting Researcher with Delft University of Technology, Delft, The Netherlands. His research interests include radar imaging, automatic target detection, and machine learning.

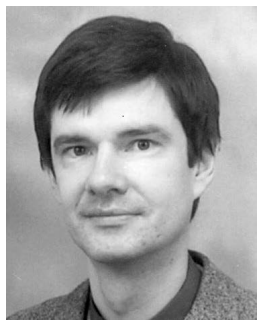

Alexander G. Yarovoy (F'15) received the Diploma degree (Hons.) in radiophysics and electronics, the Candidate Phys. \& Math. Sci. degree in radiophysics, and the Doctor Phys. \& Math. Sci. degree in radiophysics from Kharkov State University, Kharkov, Ukraine, in 1984, 1987, and 1994, respectively.

In 1987, he joined as a Researcher with the Department of Radiophysics, Kharkov State University, where he became a Professor in 1997. From 1994 to 1996 , he was with the Technical University of Ilmenau, Ilmenau, Germany, as a Visiting Researcher. Since 1999, he has been with the Delft University of Technology, Delft, The Netherlands. Since 2009, he has been the Chair of Microwave Sensing, Signals and Systems. He has authored or co-authored over 250 scientific or technical papers and 14 book chapters, and holds four patents. His current research interests include ultrawideband microwave technology and its applications (particularly radars) and applied electromagnetics (particularly UWB antennas).

Prof. Yarovoy was a recipient of the European Microwave Week Radar Award for the paper that best advances the state of the art in radar technology in 2001 (together with L. P. Ligthart and P. van Genderen) and in 2012 (together with T. Savelyev). In 2010, together with D. Caratelli, he was also a recipient of the Best Paper Award of the Applied Computational Electromagnetic Society. He has served as a Guest Editor of five Special Issues of the IEEE TRANSACTIONS and other journals. Since 2011, he has been an Associated Editor of the International Journal of Microwave and Wireless Technologies. He has served as the Chair and TPC Chair of the fifth European Radar Conference (EuRAD 08), Amsterdam, The Netherlands, as well as the Secretary of the first European Radar Conference (EuRAD 04), Amsterdam. He has also served as the Co-Chair and TPC Chair of the tenth International Conference on Ground Penetrating Radar (GPR 2004) at Delft. Since 2008, he has been the Director of the European Microwave Association. 